\title{
Liquid Bridge between Two Moving Spheres: An Experimental Study of Viscosity Effects
}

\author{
Olivier Pitois, ${ }^{1}$ Pascal Moucheront, and Xavier Chateau \\ LMSGC, 2 allée Kepler, 77420 Champs-sur-Marne, France
}

Received November 23, 1999; accepted July 17, 2000

\begin{abstract}
The effects of viscosity on the mechanical response of a liquid bridge are investigated in the case of small amounts of liquid axially strained between two moving spheres. An experimental setup allows the measurement of capillary and viscous forces exerted on the spheres as a function of the spheres separation distance and the spheres velocity. The experimental results are found to beaccurately described over a large range in spheres velocity and liquid viscosity by a simple closed-form expression. In addition, the bridge rupture distance is found to increase like the square root of the separation velocity. (c) 2000 Academic Press

Key Words: liquid bridge; viscous force; capillary force.
\end{abstract}

\section{INTRODUCTION}

A lot of work has been devoted to the study of small amounts of liquid held captive at contacts between particles (1-3). Most of the work was focused on the equilibrium shapes of static axisymmetric bridges (4-7) and the determination of forces exerted by menisci on solid surfaces, which is crucial in problems associated for example with the cohesion of moist soils $(8,9)$ or liquid-phase sintering (10). Measurements of capillary forces between two spheres showed that the maximum force appears to be at a small, but nonzero, separation distance $(3,22,24)$. Recently, the interesting work performed by Simons and coworkers for bridges of viscous binder between small spheres (20-30 $\mu \mathrm{m}$ ) seem to support this phenomenon (27-29). Squeezing of the liquid inside the gap and associated viscous (dynamic) effects arising from the measurement procedure can also be suspected (29). In the present paper, we investigate the effects of viscosity on the axial force response and the rupture distance of small liquid bridge strained between two moving spheres in perfect wetting conditions and for small Reynolds numbers.

Considering the dynamics of such liquid bridges, some work has been done in diverse fields such as the control of the thermocapillary convection, for example (11), but the determination of forces exerted by a bridge of highly viscous liquid strained between two moving spheres has received less attention. Nev-

\footnotetext{
${ }^{1}$ To whom correspondence should be addressed. LPMDI, Bât. Lavoisier, 5 Bd Descartes, Champs-sur-Marne, 77454 Marne-la-Vallée cedex 2, France. E-mail: pitois@univ-mlv.fr.
}

ertheless, viscosity effects can greatly affect the mechanical response of the bridge. In the case of a viscous binder bridging moving particles (during powder granulation processes, for example) dynamic adhesion forces developed by the bridges can be several times higher than their static counterparts (12) and the rupture distance of the bridge can be significantly increased with the liquid viscosity (13). The viscous impulse required to separate a sphere partially embedded in a layer of binder was estimated by Matthewson (14) by summing a static meniscus force and a rate-dependent viscous term. Ennis et al. (15) studied the influence of viscosity on the strength of an oscillating pendular liquid bridge between two spheres. In this pioneer paper, although general good agreement is found between experimental and theoretical results, discrepencies appear when the bridge volume is small and the gap is large. The aim of this paper is to measure the static and dynamic forces exerted by a bridge of Newtonian liquid with a sufficient precision to allow an accurate comparison of the results with theoretical predictions. Theoretical considerations are given in the next part of the paper. The experimental setup is presented in the third part and the results obtained are presented and discussed in part four, before we conclude.

\section{THEORY}

\subsection{Capillary Adhesion}

For small amounts of liquid, gravity effects can be neglected, and the static attractive force due to the meniscus can be expressed as the sum of the capillary force and the axial component of the surface tension force acting on the spheres of radius $R(9)$,

$$
F_{\text {Cap }}=2 \pi R \sigma \sin \phi \sin (\phi+\theta)-\Delta P \cdot \pi R^{2} \sin ^{2} \phi,
$$

where $\phi$ is the half-filling angle, $\theta$ is the solid/liquid contact angle, and $\sigma$ is the surface tension. The difference in hydrostatic pressure across the interface $(\Delta P)$ is related to the local mean curvature $\Gamma$ and to the surface tension by the Young-Laplace equation,

$$
\Delta P=\sigma \cdot 2 \Gamma,
$$




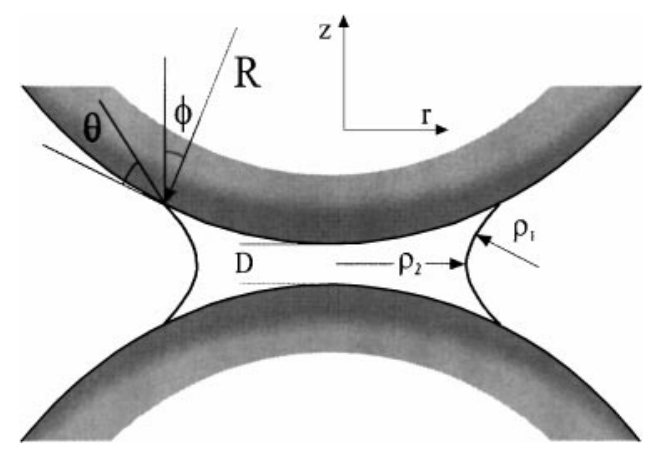

FIG. 1. Liquid bridge between two spheres.

where $\Gamma$ can be expressed as a function of the cylindrical coordinates of the radial profile of the liquid-gas interface (see Fig. 1):

$$
2 \Gamma=\frac{1}{r\left[1+(d r / d z)^{2}\right]^{1 / 2}}-\frac{d^{2} r / d z^{2}}{\left[1+(d r / d z)^{2}\right]^{3 / 2}} .
$$

Equation [2] can be solved analytically in terms of elliptic integrals (16). One alternative to exact methods is to consider a circular approximation for the meridian profile of the interface. This toroïdal approximation for the meniscus shape leads to an interface of nonconstant mean curvature, so that the resulting attractive force is not constant along the $z$ axis and there are several ways of evaluating $F_{\text {Cap }}$. One of them, the "gorge method," gives total forces within $10 \%$ of those obtained by means of an exact numerical technique (17) and leads to the simple closed-form expression,

$$
\begin{aligned}
F_{\text {Gorge }} & =\pi \rho_{2}^{2} \Delta P+2 \pi \rho_{2} \sigma=\pi \sigma \rho_{2}\left[1+\frac{\rho_{2}}{\rho_{1}}\right] \\
\rho_{1} & =\frac{D / 2+R(1-\cos \phi)}{\cos (\phi+\theta)} \\
\rho_{2} & =R \sin \phi-[1-\sin (\phi+\theta)] \rho_{1}
\end{aligned}
$$

where $\rho_{1}$ and $\rho_{2}$ are respectively the radius of the meridian profile and the radius at the neck (see Fig. 1). In addition, assuming that $R \gg \rho_{2} \gg \rho_{1}$ and $D \ll 2 \rho_{1} \cos \theta$, a simplified expression can be derived for $F_{\text {cap }}(18)$ :

$$
F_{\text {Cap }} \approx 2 \pi R \sigma \cos \theta \cdot\left[1-\frac{D}{2 \rho_{1} \cos \theta}\right]
$$

Assuming now that the liquid bridge is approximately cylindrical (flat profile) and that its volume is given by

$$
V=\int_{0}^{b} 2 \pi r H(r) d r=\frac{\pi R}{2}\left[H^{2}(b)-D^{2}\right],
$$

where $b$ is the radius of the wetted area and $H(r)=D+r^{2} / R$, an expression is obtained for the force as a function of the bridge volume and the separation distance:

$$
F_{\text {Cap }} \approx 2 \pi R \sigma \cos \theta \cdot\left[1-\frac{1}{\sqrt{1+\frac{2 V}{\pi R D^{2}}}}\right] .
$$

This expression was earlier proposed by Maugis (19) within the framework of rupture mechanics theories. Nevertheless, no quantitative comparison of this latter expression with experimental or theoretical results has been performed.

Note also that, in the presence of a gravitational field, the pressure variation as a function of height above a reference plane has to be considered in accordance with Pascal's law. In this case, numerical evaluation procedures are required (20-22).

\subsection{Viscous Forces}

The mechanics of thin liquid films are described by the wellknown Reynolds equation, which relates the pressure $P$ generated in the liquid to the relative displacement of the two solid surfaces (23):

$$
\frac{d}{d r}\left[r H^{3}(r) \frac{d P(r)}{d r}\right]=12 \eta r \frac{d D}{d t} .
$$

Integrating twice Eq. [8], an expression can be derived for the viscous force acting on the spheres (assuming an infinite liquid):

$$
F_{\mathrm{vis}}=-\frac{3}{2} \pi \eta R^{2} \frac{1}{D} \frac{d D}{d t} .
$$

In the case of a finite volume of liquid (cylindrical bridge), Matthewson (14) proposed a correction coefficient to Eq. [9], which becomes

$$
F_{\text {vis }}=-\frac{3}{2} \pi \eta R^{2}\left[1-\frac{D}{H(b)}\right]^{2} \frac{1}{D} \frac{d D}{d t} .
$$

Note that this latter expression can be related to the bridge volume using Eq. [6].

\section{EXPE RIMENTAL}

\subsection{Experimental Arrangement}

An apparatus was constructed for measuring the resultant vertical forces exerted by a viscous liquid meniscus strained between two moving spheres. The main part of this apparatus is schematically shown in Fig. 2. The meniscus is formed between two polished ruby spheres of radius $R=4 \mathrm{~mm}( \pm 1 \mu \mathrm{m})$. The upper one is bolted under the platten of a counter-reaction scale (Sartorius MDRA200), which allows measurement of the vertical force applied to the sphere without displacement of it. Forces can be measured in the range $-1 / 5 \mathrm{~N}$ with a precision of $10 \mu \mathrm{N}$. The other sphere is bolted to a metallic rod guided along the vertical axis by two planar springs. The rod can be moved up 


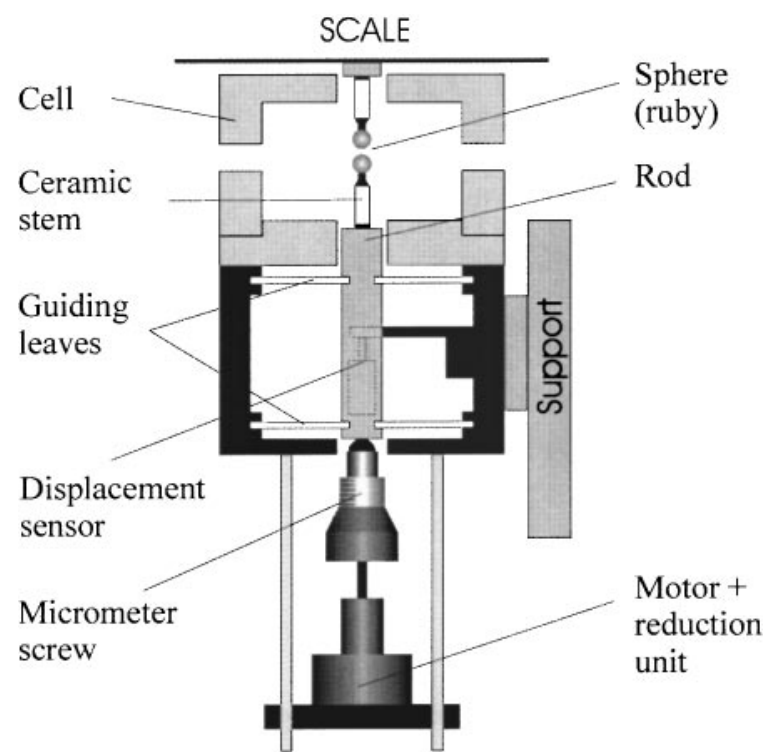

FIG. 2. Experimental liquid bridge apparatus.

and down by means of a motor-driven differential micrometer screw allowing the increase or decrease of the spheres separation distance at a constant velocity. The separation distance can be adjusted in the range $0-1 \mathrm{~mm}$ and the displacement velocities can be varied from 0.01 to $15 \mu \mathrm{m} / \mathrm{s}$. A displacement sensor tracks the position of the rod with a precision of about $\frac{1}{4} \mu \mathrm{m}$.

The element described above stands in a large thermostated cell (generally $T=22 \pm 0.2^{\circ} \mathrm{C}$ ). A small thermostated chamber can be used to maintain locally the spheres at a temperature significantly different from the ambient temperature. A lens and a camera (connected to a computer) allow us to save images of the contact region.

\subsection{Materials}

Bridge materials and related properties $\left(25^{\circ} \mathrm{C}\right)$ are presented in Table 1 . The angles of contact of oils with the ruby spheres were found to be in the range $0^{\circ}-10^{\circ}$. The value of $\theta$ will be taken equal to $10^{\circ}$ in every theoretical evaluation.

\subsection{Experimental Procedure}

First, we measure the position for which contact between spheres is detected (nonzero force detected). This reference position allows us to pull the spheres apart with a known separation distance $(D)$. An amount of liquid is then inserted into

TABLE 1

Properties of the Liquids $U$ sed during the Experiments

\begin{tabular}{lcc}
\hline \multicolumn{1}{c}{ Liquids } & Surface tension $(\mathrm{mN} / \mathrm{m})$ & Viscosity $(\mathrm{mPa})$ \\
\hline 1 (PDMS Oil) & 21 & 0.1 \\
2 (PDMS Oil) & 21 & 100 \\
3 (Brookfield Oil) & 19.5 & 400 \\
\hline
\end{tabular}

the contact region with a syringe. Images of the contact region before and after the formation of the meniscus and some image processing allow the determination of the bridge volume with a precision of about 5\%. Several accomodation cycles of spheres approach and separation are generally performed before starting the measurements to obtain a perfect axisymmetric bridge.

\section{RESULTS AND DISCUSSION}

\subsection{Capillary Regime: $F_{\text {cap }} \gg F_{\text {vis }}$}

Measurements have been performed with liquid 1 during the increase of $D$ at a low rate: $v=0.01 \mu \mathrm{m} / \mathrm{s}$. The results for the attractive capillary force are presented in Figs. $3 \mathrm{a}$ and $3 \mathrm{~b}$ for two bridge volumes: $V \approx 0.05$ and $V \approx 1.5 \mu$ l. The values given by expressions Eq. [4] and Eq. [7] as well as curves corresponding to
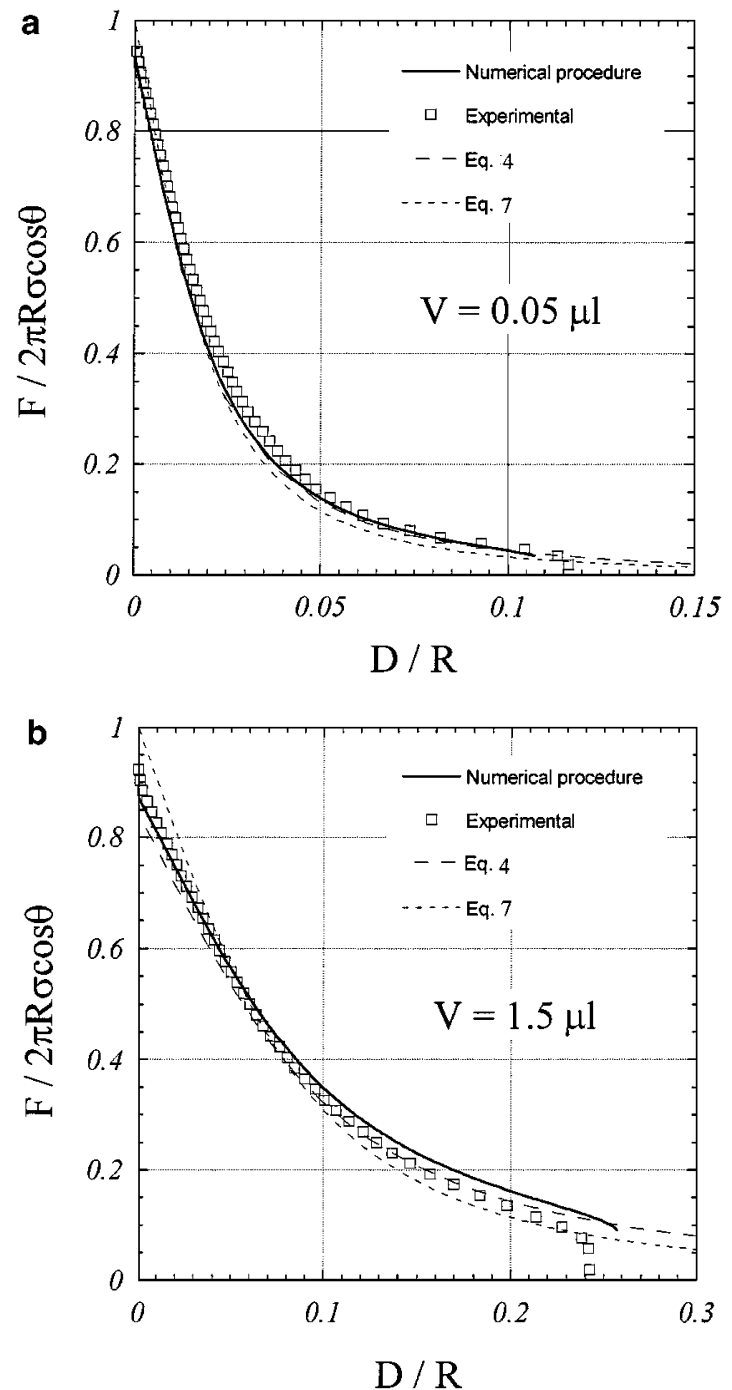

FIG. 3. Comparison of experimental results with theoretical values for the attractive capillary force exerted by a liquid bridge (liquid 1) between two spheres (a) Bridge of volume $V=0.05 \mu$ l. (b) $V=1.5 \mu 1$. 
an exact numerical resolution taking gravity effects into account (see Ref. 22 for more details about the numerical procedure) are also plotted.

It can be seen in Fig. 3 that, in contrast to the results reported by several authors $(3,22,24,28,29)$, the maximum of force is reached for $D=0(F \approx 0.5 \mathrm{mN})$, and then the force decreases as the separation distance increases, until the bridge breaks. The present results are in good accordance with theoretical predictions, although small discrepancies with numerical evaluation stem from the imprecision on volume measurements. The curves are calculated for the volume measured and no attempt to fit this parameter has been done. It can be seen that for the smallest liquid volume all prediction methods give values very close to the experimental one. From a practical point of view, note the interest of using Eq. [7] in evaluating the capillary force exerted by small amounts of liquid with low contact angles. In contrast to the two other methods, some discrepancies are observed for the largest liquid volume, especially at small separation distances.

Note also the good accuracy of the numerical method in predicting the bridge rupture distance. The end of the black lines indicates that no equilibrium configuration does exist for higher separation distances (7).

\subsection{Viscous Regime: $F_{\text {cap }} \ll F_{\text {vis }}$}

The repulsive force exerted by a highly viscous liquid bridge (liquid 3) has been measured during the spheres approach. In the present situation, viscous effects are about $10^{6}$ times higher than those in the previous case. Some results are presented in Fig. 4 for a bridge volume $V=2.2 \mu \mathrm{l}$ and for several approach velocities. The values obtained with Eq. [10] for the corresponding spheres velocities are also plotted, taking $\eta=410 \mathrm{~Pa}$. Good

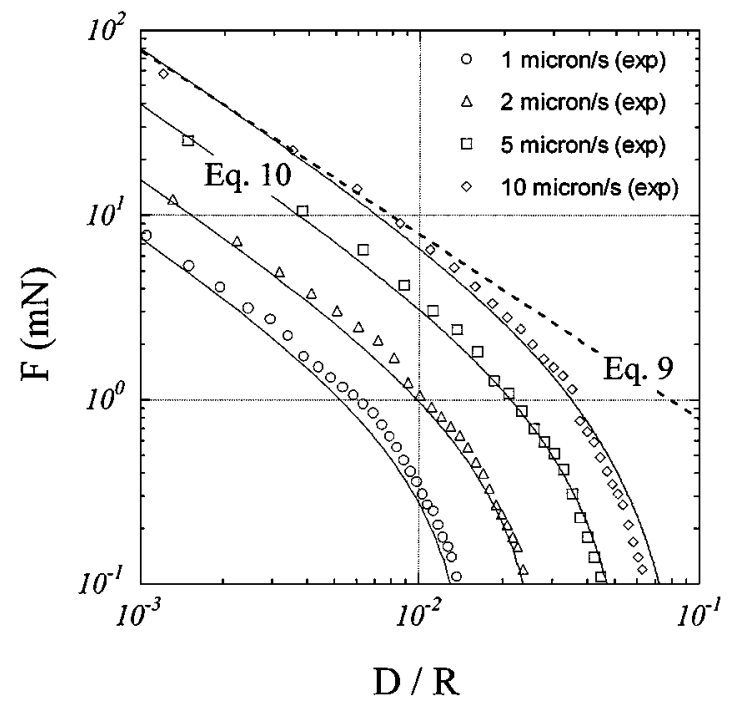

FIG . 4. Comparison of experimental results with theoretical values for the repulsive viscous force exerted by a liquid bridge (liquid 3, $V=2.2 \mu \mathrm{l}$ ) between two spheres and for several sphere velocities.

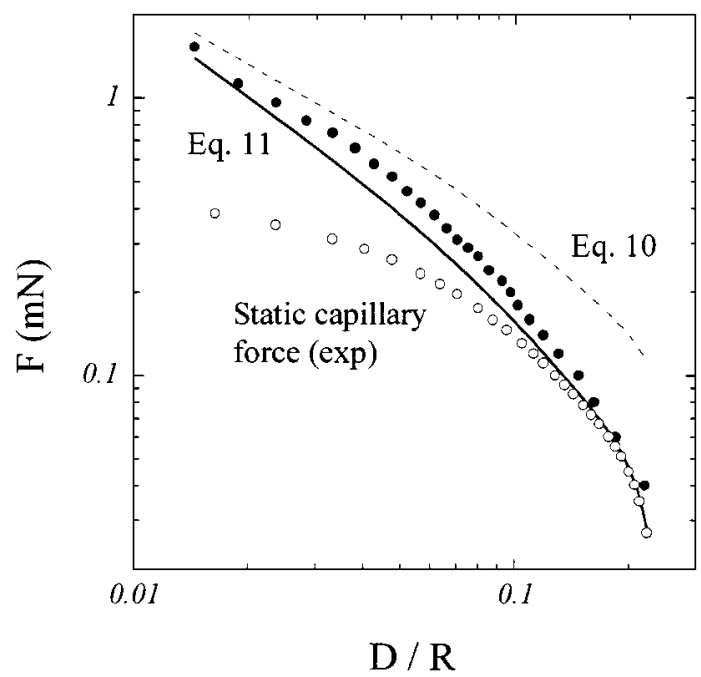

FIG. 5. Dynamic attractive force exerted by a liquid bridge (liquid $2, V=$ $1.1 \mu \mathrm{l})$ measured between two spheres separating at a constant velocity $(v=$ $5 \mu \mathrm{m} / \mathrm{s}$ ). Measurements corresponding to the static capillary force are also presented.

accordance is observed between theoretical and experimental values, accounting for the validity of Eq. [10] and the lubrication assumptions. Note that, in this experiment, whatever the separation distance and the spheres velocity, the order of magnitude of the Reynolds number is $R e \approx w b^{2} v / \eta D \ll 1$ where $w$ is the volumic mass of the liquid. Note also that for the smallest values of $D / R$ the influence of the interface becomes very weak, so that values given by Eqs. [9] and [10] are very close (straight lines are observed).

\subsection{Intermediate Regime: $F_{\text {cap }} \approx F_{\text {vis }}$}

Results for the attractive force measured during the increase of the separation distance are presented in Fig. 5 in the case of a sphere velocity $v=5 \mu \mathrm{m} / \mathrm{s}$ and a volume $V=1.1 \mathrm{~mm}^{3}$ of liquid 2 . The values corresponding to the static capillary force (determined for $v=0.01 \mu \mathrm{m} / \mathrm{s}$ ) and to expression Eq. [10] are also presented. It can be seen that the viscous forces dominate for the smallest values of $D$ whereas interactions are dominated by capillary forces for the highest separation distances. Note in particular that the maximum dynamic attractive force measured is about 3 times higher than the corresponding static one.

A more interesting situation is presented in Fig. 6: the force is measured during the spheres approach, for $v=10 \mu \mathrm{m} / \mathrm{s}$. In this case, it is observed that the force is attractive for large separation distances (capillary regime) and repulsive for the smallest one (viscous regime). For intermediate values of $D$, a transitional regime is observed. Note in particular the presence of a maximum $\left(F_{\max }\right)$ for the attractive force.

Following the approach proposed by Ennis et al., the total force is now evaluated by summing a static capillary term (Eq. [4]) and a lubrication term (Eq. [9]). It can be seen in Figs. 5 and 6 that this expression does not lead to values compatible with 


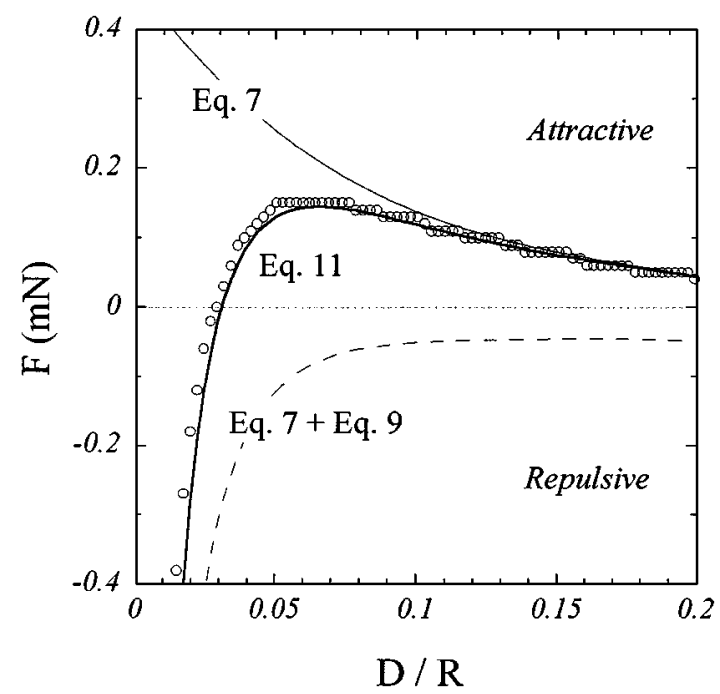

FIG. 6. Dynamic force exerted by a liquid bridge (liquid 2, $V=1.1 \mu \mathrm{l}$ ) measured between two spheres approaching at a constant velocity $(v=$ $10 \mu \mathrm{m} / \mathrm{s})$.

the present experimental results. In particular (in Fig. 6), a repulsive force is predicted for all separation distances. We propose to sum Eq. [7] and Eq. [10], leading to the simple closed-form expression:

$$
\left.\begin{array}{c}
F_{\text {tot }}=F_{\text {Cap }}+F_{\text {vis }}=2 \pi R \sigma \cos \theta \cdot X_{\mathrm{V}}+\frac{3}{2} \pi \eta R^{2} \frac{1}{D} \frac{d D}{d t} \cdot X_{\mathrm{V}}^{2} \\
\text { with } X_{\mathrm{V}}=1-\left[1+\frac{2 V}{\pi R D^{2}}\right]^{-1 / 2} \cdot
\end{array}\right\}
$$

Equation [11] is analogous to the one proposed by Matthewson (14), but in the present case, $F_{\text {tot }}$ is directly expressed as a function of the bridge volume and the separation distance. Note that the correction factor $\left(X_{\mathrm{V}}^{2}\right)$ introduced in Eq. [11] significantly reduces the viscous contribution in the case of small liquid volumes and large gap. Taking $D^{*}=0.023$, for example, $X_{\mathrm{V}}^{2}$ equals $0.1,0.5$, and 0.8 for $V^{*}=0.001,0.01$, and 0.1 , respectively. The values obtained with Eq. [11] are plotted in Figs. 5 and 6. Good accordance is observed with experimental results, in particular, in the case of approaching spheres, where the transitional regime is accurately described. Experimental and predicted values for $F_{\text {max }}$ are plotted in Fig. 7 as a function of $v$. It can be seen that the maximal attractive force measured during the spheres approach is well described by Eq. [11]. During experiments, it was observed that the interface profile could be significantly different from the corresponding equilibrium static one. Nevertheless, this particular behavior occurs as the separation distance is small so that the capillary contribution is generally low with regard to the viscous one.

Another interesting effect of the liquid viscosity on the bridge behavior concerns the bridge rupture distance. Beyond a critical separation distance $\left(D_{\text {rupt }}^{\mathrm{S}}\right)$, the amount of liquid cannot bridge the two spheres anymore, so that it breaks into two parts. It has been shown (17) that this rupture distance is related to the bridge volume and the contract angle by the approximate relation

$$
D_{\text {rupt }}^{\mathrm{S}} \approx\left[1+\frac{\theta}{2}\right] \cdot V^{1 / 3}
$$

In the presence of viscosity effects, the bridge rupture does not occur instantaneously during the spheres separation process, so that the dynamic bridge rupture distance $\left(D_{\text {rupt }}^{\mathrm{d}}\right)$ can be significantly larger than the corresponding static one. This was qualitatively observed during experiments on liquid bridges between two spheres (13) and quantitatively in the particular case of slender liquid bridges (of moderate viscosity) stretched between two disks moved apart at a relatively high speed (26) (in this case the triple line is fixed on the disks perimeter).

Measurements of $D_{\text {rupt }}^{\mathrm{d}}$ in the case of small amounts of highly viscous liquid (liquid 2) stretched between two spheres pulled apart at constant velocities have been performed. The volume of liquid was chosen small enough $(V=0.5 \mu \mathrm{l})$ for the corresponding rupture distance to be smaller than the maximum separation gap $(1 \mathrm{~mm})$ for all separation velocities. The evolution of the liquid bridge rupture distance, expressed by

$$
\Delta D_{\text {rupt }}=\frac{D_{\text {rupt }}^{\mathrm{d}}-D_{\text {rupt }}^{\mathrm{S}}}{D_{\text {rupt }}^{\mathrm{S}}},
$$

is plotted in Fig. 8 as a function of $v$.

The results clearly indicate that the rupture distance increases as the sphere velocity increases: for $v=10 \mu \mathrm{m} / \mathrm{s}, D_{\text {rupt }}^{\mathrm{d}}$ is $20 \%$ larger than the corresponding $D_{\text {rupt }}^{\mathrm{S}}$. A power law exponent can

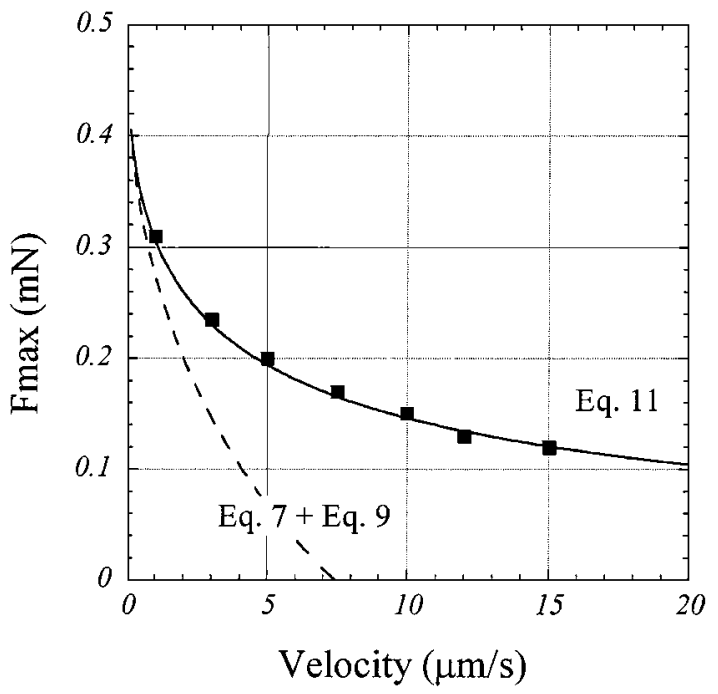

FIG 7. Maximum attractive force (bridge of liquid $2, V=1.1 \mu 1$ ) measured during spheres approach as a function of the sphere velocity. 


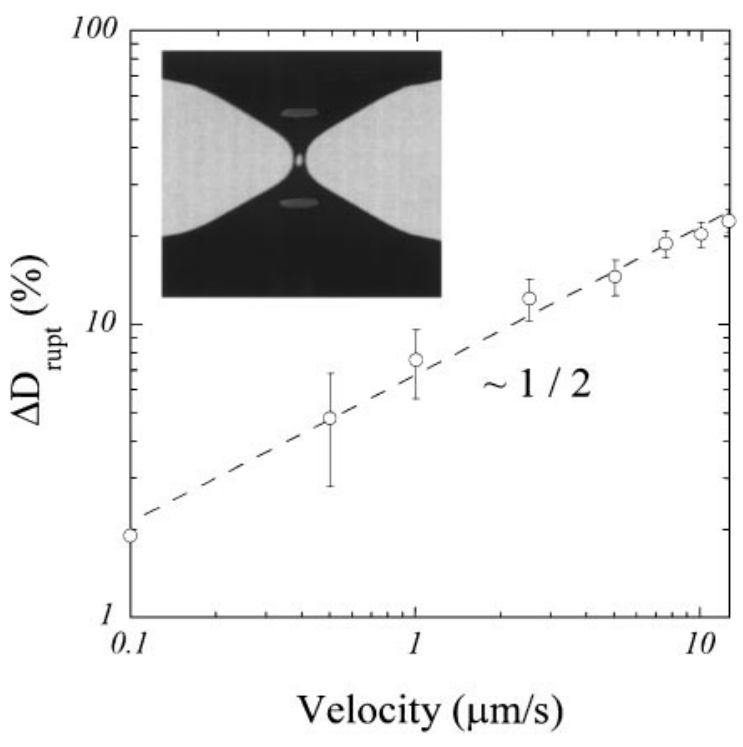

FIG. 8. Increase of the bridge rupture distance (liquid $2, V=0.5 \mu \mathrm{l}$ ) as a function of the sphere velocity. The bridge rupture is illustrated by the image presented in the insert (the bright point in the liquid filament is an optical artefact).

be identified over 2 decades of the velocity:

$$
\Delta D_{\text {rupt }} \propto v^{1 / 2}
$$

This power law seems to be hardly predictable through simple theoretical considerations. In fact, the rupture mechanism is associated with a complex interface deformation process. The image presented in Fig. 8 clearly shows that the bridge rupture proceeds in the formation of a thin liquid filament linking the two volumes of liquid resting on the spheres. Note also the high value of the apparent dynamic solid/liquid contact angle and the particular conical shape of the liquid remaining on each sphere.

\section{CONCLUSION}

The total axial force exerted by a liquid bridge strained between two spheres has been measured with sufficient resolution to allow an accurate comparison of the results with theoretical predictions. In the quasi-static regime, capillary forces have been measured and the results have been found to be in good accordance with the values given by a numerical procedure and by approximate expressions. In contrast to several published experimental results, the maximum of force has been found for a zero separation distance. Besides, in the case of small amounts of liquid $\left(V^{*}<0.01\right)$, experimental values were well described by a simple expression (Eq. [7]). In the case of additional viscous effects, a rate-dependent term has to be taken into account for evaluating the total force. The expression proposed by Ennis et al. had been found to fail in predicting the total force when capillary and lubrication forces are of the same order of magnitude. We proposed a modified analytical expression (Eq. [11]), directly related to the bridge volume. Good accordance of the experimental results with Eq. [11] has been observed over a large range of liquid viscosity and sphere velocity. The bridge rupture distance was also measured. It has been shown that viscous effects contribute to the increase in the rupture distance, which was found to vary like the square root of the separation velocity.

\section{ACKN OWLEDGMENT}

The authors would like to acknowledge the technical contributions made by J.-P. Guilbaut (LMSGC).

\section{REFERENCES}

1. Boucher, E. A., Evans, M. J. B., and McGarry, S., J. Colloid Interface Sci. 89, 154-165 (1982).

2. Erle, M. A., Dyson, D. C., and Morrow, N. R., AIChE J. 17, 115-121 (1971).

3. Mason, G., and Clark, W. G., Chem. Eng. Sci. 20, 859-866 (1965).

4. Bayramli, E., Abou-obeid, A., and Van de ven, T. G. M., J. Colloid Interface Sci. 116, 503-510 (1987).

5. De Bisschop, F. R. E., and Rigole, W. J. L., J. Colloid Interface Sci. 88, 117-128 (1982).

6. Mehrotra, V. P., and Sastry, K. V. S., Powder Technol. 25, 203-214 (1980).

7. Saez, A. E., and Carbonell, R. G., J. Colloid Interface Sci. 140, 408-418 (1990).

8. Haines, W. B., J. Agric. Sci. 15, 529-543 (1925).

9. Fisher, R. A., J. Agric. Sci. 16, 492-505 (1926).

10. Heady, R. B., and Cahn, J. W., Met. Trans. 1, 185-189 (1970).

11. Anilkumar, A. V., Grugel, R. N., Shen, X. F., Lee, C. P., and Wang, T. G., J. Appl. Phys. 73, 4165-4170 (1993).

12. Adams, M. J., and Perchard, V., Inst. Chem. Eng. Symp. Ser. 91, 147-160 (1984).

13. Mazzone, D. N., Tardos, G. I., and Pfeffer, R., Powder Technol. 51, 71-83 (1987).

14. Matthewson, M. J., Philos. Mag. A 57, 207-216 (1988).

15. Ennis, B. J., Li, J., Tardos, G., and Pfeffer, R., Chem. Eng. Sci. 45, 30713088 (1990).

16. Orr, F. M., Scriven, L. E., and Rivas, A. P., J. Fluid Mech. 67, 723-742 (1975).

17. Lian, G., Thornton, C., and Adams, M. J., J. Colloid Interface Sci. 161, 138-147 (1993).

18. Fisher, L. R., and Israelachvili, J. N., Colloids Surf. 3, 303-319 (1981).

19. Maugis, D., J. Adhesion Sci. Technol. 105-134 (1987).

20. Bayramli, E., Abou-obeid, A., and Van de ven, T. G. M., J. Colloid Interface Sci. 116, 490-502 (1987).

21. Coriel, S. R., Hardy, S. C., and Cordes, M. R., J. Colloid Interface Sci. 60, 126-136 (1977).

22. Mazzone, D. N., Tardos, G. I., and Pfeffer, R., J. Colloid Interface Sci. 113, 544-556 (1986).

23. Cameron, A., "The Principles of Lubrication." Longmans, Green, New York, 1964.

24. Hotta, K., Takeda, K., and Iinoya, K., Powder Technol. 10, 231-242 (1974).

25. Lian, G., Thornton, C., and Adams, M. J., in "Powders and Grains 93" (Thornton, Ed.), pp. 59-64. Balkema, Rotterdam, 1993.

26. Zhang, X., Padgett, R. S., and Basaran, O. A., J. Fluid Mech. 329, 207-245 (1996).

27. Fairbrother, R. J., and Simons, S. J. R., Part. Part. Syst. Charact. 15, 16-20 (1998).

28. Simons, S. J. R., and Fairbrother, R. J., Powder Technol. 110, 44-58 (2000).

29. Fairbrother, R. J., and Simons, S. J. R., in "World Congress on Particle Technology 3," paper 79. Institution of Chemical Engineers, New York, 1998. 